\title{
Corea y el proceso de integración centroamericana: desafíos y oportunidades
}

\author{
Seok-Hwa HONG
}

Recepción: 28-03-2016 / Aceptación: 12-05-2016

\section{Resumen}

El presente artículo toma como punto central el estudio del proceso de integración centroamericana, desde sus inicios hasta su estado actual, identificando los principales obstáculos que ha enfrentado los países que conforman el sistema de integración centroamericana (SICA), particularmente los países centroamericanos, por alcanzar una unidad completa. En este sentido, se analiza el papel que ha desempeñado la República de Corea en contribuir a dicha integración; su relación con los países centroamericanos y algunas estrategias que deberían implementarse para fortalecer las relaciones políticas, económicas y de cooperación entre ambas partes.

Palabras Claves: Integración, Centroamérica, SICA, Corea

\begin{abstract}
The present article takes as its central point the study of Central American integration process, from its beginnings to its current state, identifying the main obstacles that the countries of the Central American Integration System (SICA), particularly the Central American countries, have faced to achieve a complete unity. In this regard, the role played by the Republic of Korea to contribute to this integration is analyzed; its relationship with the Central American countries and some strategies that should be implemented to strengthen political, economic and cooperative relations between both sides.
\end{abstract}

Keywords: Integration, Central America, SICA, Korea

\section{Introducción}

La subregión centroamericana cuenta con uno de los procesos de integración más dinámicos y profundos de América Latina (CEPAL, 2014, p. 7).

Desde la suscripción del Tratado General de Integración Económica Centroamericana, en Managua, en 1960, el proceso de integración centroamericana ha experimentado ciertos avances que han permitido un mejor intercambio comercial entre los países miembros y ha constituido el preámbulo para alcanzar su fin último: la unidad y desarrollo integral como subregión.
Para tal efecto, se han suscrito diversos acuerdos a nivel regional, y se han celebrado Reuniones de Presidentes y Ministros, a fin de determinar las temáticas a incluir en las agendas de trabajo y las políticas a definir para superar las brechas existentes entre los países de la región.

En el presente artículo se hace un recorrido hacia los orígenes del proceso de integración centroamericana hasta llegar al estado actual de la integración, identificando cuáles son los sectores más vulnerables en el proceso de integración y qué desafíos ha enfrentado y/o tiene que superar la región para lograr su unidad. 
En este sentido, se estudia el papel que ha jugado la República de Corea, haciendo una breve referencia a los aportes que ha brindado el país asiático hacia el SICA y sus países miembros; y de qué manera podría este país contribuir de manera más efectiva en el proceso de integración y desarrollo de los países de la región.

Para el presente artículo se tomó como referencia los instrumentos jurídicos constitutivos delSICA, algunas publicaciones elaboradas por organismos internacionales en el tema de integración, como la CEPAL y la OIT; así como, libros y revistas publicados por expertos en el tema.

\section{Perspectiva histórica del proceso de integración centroamericana}

A lo largo de la historia los países centroamericanos han realizado esfuerzos para alcanzar su integración. Así, desde la época colonial pueden percibirse los espíritus unionistas de Centroamérica, cuyos países una vez obtenida su independencia de España en 1821, deciden conformar la llamada Provincias Unidas de Centroamérica, durante reunión en el Congreso Constituyente en Guatemala, el 1 de Julio de 1823, convirtiéndose éste en el primer intento de integración.

A partir de ese momento se adopta la idea de constituir una federación centroamericana, la cual queda jurídicamente contemplada en la Constitución de la Federación Centroamericana, promulgada en noviembre de 1824. Desgraciadamente, este intento de integración no tuvo éxito debido a los vacíos y ambigüedades contempladas en la Carta Magna con relación al funcionamiento del poder federal y las voluntades de los cinco Estados miembros.
Como señala Cardenal (1996), “esta ambigüedad de poderes y jurisdicciones hizo crisis al plantear el financiamiento del presupuesto federal. Los ingresos debían salir de los Estados, pero estos siempre estuvieron muy necesitados de dinero. A ello hay que agregar que tanto el gobierno federal como el estatal tenían poderes Ejecutivo, Legislativo y Judicial" (p. 235).

En el período comprendido entre 1823 y 1840 se pudo hablar de una federación centroamericana pero esta llegó a su fin con causas muy variadas como una falta de integración a nivel regional, la desigual distribución de la población, el localismo, los errores de la Constitución federal, problemas de carácter ideológico entre liberales y conservadores, y la falta de una base económica sólida frustraron el intento de lo antes llamado el Reino de Guatemala, para que se mantuviera unido como una república federal (Cardenal. 1996: 138).

En 1847, la amenaza extranjera en Nicaragua, dio origen a un intento más por la unión de los países, con un gobierno provisional, cuyo objetivo fundamental sería el de "prestarse recíprocamente auxilio para la conservación de su independencia y soberanía, y consecutivamente a auxiliarse mutuamente para conservar las formas de gobierno popular y representativo"1.

A principios del siglo $X X$ se siguieron dando intentos unificadores, tales como el Pacto de Corinto o Tratado de Paz y Arbitraje Obligatorio, de 1902, el cual fue suscrito por Honduras, El Salvador, Costa Rica y Nicaragua, y que contemplaba la creación de un arbitraje obligatorio y de un tribunal centroamericano. No obstante, dicho Tratado quedó inoperante en 1907, debido entre otras cosas, al estallido

1 OIT. (1999). El Proceso de Integración Centroamericana en Perspectiva. Recuperado de www.ilo.org/public/spanish/region/ampro/ mdtsanjose/worker/integ_ca/final.htm 
de una guerra entre Guatemala, Honduras y El Salvador en 1906.

Igualmente, en 1921 surge una nueva manifestación de integración, mediante la creación de la República Tripartita, promovida por el entonces Presidente de Guatemala Carlos Herrera, a través del Tratado de la Unión Centroamericana, suscrito por Guatemala, Honduras y El Salvador; en virtud del cual las tres naciones declaraban formar la República de América Central, con capital en Tegucigalpa, y reservando un puesto tanto para Nicaragua, como para Costa Rica, para que tan pronto como se decidieran apoyar aquella unión formasen también parte de la misma. Según este acuerdo, cada Estado conservaría su carácter particular y la Federación únicamente aplicaría en las Relaciones Exteriores y los asuntos de mayor interés general. No obstante, debido a la Revolución ocurrida en Guatemala, que trae consigo el derrocamiento del Presidente Herrera, este Tratado pierde su validez.

No es sino hasta 1951 que nace la Carta de la Organización de Estados Centroamericanos (ODECA), la cual persigue alcanzar una integración regional que promoviera e impulsara la recíproca cooperación económica, social y técnica, para lo cual también se crea una Corte de Justicia Centroamericana y un Consejo Económico.

Posteriormente, en 1960, los países de Guatemala, El Salvador, Honduras y Nicaragua firman el Tratado General de Integración, el cual da origen a la constitución de un Mercado Común Centroamericano (MCC), con la consecuente creación de un arancel común de libre comercio centroamericano y un arancel común externo; así como, el establecimiento de la Secretaría Permanente del Tratado General de Integración Económica Centroamericana (SIECA) y el Banco Centroamericano de Integración Económica (BCIE). Dicho Tratado fue posteriormente suscrito por Costa Rica en 1962.
El Mercado Común Centroamericano se formó con el fin de lograr una rápida industrialización de la región, la cual estaba sujeta al intercambio mutuo de los beneficios netos que se esperaban como resultado de su creación. A su vez, se esperaba que la industrialización trajera consigo un mayor nivel de ingresos y contribuyera a disminuir el grado de dependencia del resto del mundo, lo cual provocó una redistribución de los recursos para lo cual era necesario contar con nuevos instrumentos normativos (Bulmer. 1998:24).

En este sentido, en la segunda mitad de la década de los 80's y principios de 1990, y ante el estancamiento en el proceso de integración sufrido en los años 70's, producto de las guerras civiles a lo interno de varios países de la región; se efectuaron cumbres presidenciales, a fin de elaborar una estrategia de pacificación y resolución de conflictos para la subregión, retomando las laborares encaminadas a la integración centroamericana.

Al respecto, se crearon instituciones encaminadas a fortalecer el ámbito político de la integración, tales como las Reuniones de Presidentes y el Parlamento Centroamericano.

Más tarde, en 1991, con la suscripción del Protocolo de Tegucigalpa se da un gran avance para la unión centroamericana, ya que con este Tratado se crea el Sistema de Integración Centroamericana, que sustituye a la ODECA, como un nuevo marco jurídico-político, para lograr la integración centroamericana en todos los aspectos (económicos, políticos, sociales, culturales y ambientales).

Por medio de este Sistema se establecen nuevas entidades a nivel regional, y se amplían las funciones de órganos ya establecidos, como es el caso de la Corte Centroamericana de Justicia. 


\section{Panorama actual del Sistema de Integración Centroamericana}

Desde la creación del SICA en 1991, el proceso de integración centroamericana ha logrado grandes avances en su misión unificadora. Sus esfuerzos le han permitido a la subregión centroamericana obtener una mayor presencia y reconocimiento en el ámbito internacional, pasando de ser un mero espectador, al actor principal en la negociación de acuerdos comerciales, como el Tratado de Libre Comercio DR-CAFTA y el Acuerdo de Asociación con la Unión Europea (AdA).

No obstante, a pesar de los avances logrados, y teniendo en cuenta el actual contexto económico mundial, se hace necesario fortalecer los lazos de unidad centroamericana y desarrollar estrategias que le permitan hacer frente de manera más efectiva a los retos de un mercado libre mucho más amplio (Acevedo, R. 2015).

Es necesario superar las discrepancias de orden político, y establecer marcos nacionales $\mathrm{y}$ regionales que regulen y velen por la existencia de un balance entre los intereses de cada país miembro y los intereses como subregión.

Así mismo, deben erradicarse las desigualdades que existen en el sector educativo, en tecnología, en comercio, entre otros; impulsar el establecimiento de una verdadera unión aduanera, la armonización de normas y disposiciones comunes que rijan el comercio de bienes y servicios, entre otros. A continuación se abordan los diversos aspectos que incluye el proceso de integración centroamericana.

\subsection{Integración política}

Como señala Miranda (2010), citando a Bloch (2002), "la integración política es el proceso por el cual los actores políticos de varios ordenamientos nacionales diversos son persuadidos para desplazar sus lealtades, expectativas y actividades políticas hacia un nuevo centro, cuyas instituciones poseen o reclaman jurisdicción sobre los Estados nacionales preexistentes" (Miranda, 2010: 30).

Esta integración política no supone necesariamente una fusión de parte de los Estados que la componen; sino más bien, y como es el caso de la Unión Europea y del SICA, implica la creación de una nueva entidad o autoridad que vele por el cumplimiento de los objetivos de esa unidad, plasmados en un instrumento jurídico que legitime su actuar.

En el caso particular de Centroamérica, encontramos dicho instrumento esencialmente en el Protocolo de Tegucigalpa y sus enmiendas, el cual establece la creación del SICA (nueva entidad o autoridad), con sus diversos órganos, para la "realización de la integración centroamericana, como una región de paz, libertad, democracia y desarrollo" (Artículo 3 P.T.). Así mismo, en el seno del SICA, el órgano encargado de promover la integración política de la región es la Reunión de Presidentes, el cual es el órgano supremo, que dentro de sus atribuciones tiene la función de "definir y dirigir la política centroamericana, estableciendo las directrices sobre la integración de la región" (Artículo 15 P.T.).

Dichaintegración política seevidencia a través de las diversas políticas implementadas por los países centroamericanos en aras de una unión aduanera, migratoria; la homologación delos sistemas educativos; la armonización de la legislación penal; políticas para combatir y erradicar el hambre y la desnutrición; cambio climático; seguridad ciudadana, entre otros.

En este sentido, considero que la integración política es sumamente importante, porque constituye el primer paso a la unidad regional. Sólo habiendo una voluntad clara 
de las partes involucradas en promover la unidad regional, es que la entidad que establezcan para tal fin (en este caso el SICA) tendrá las atribuciones suficientes y contará con órganos debidamente facultados para poder desarrollar las políticas que permitan la integración, sea esta económica, ambiental, social o de cualquier otra índole.

En tal sentido, es necesario limar las asperezas políticas que puedan existir entre los miembros, como lamentablemente se evidenció entre Nicaragua y Costa Rica, y que tuvo como consecuencia la salida de este último de los foros políticos del SICA; y se deben tomar medidas consensuadas que eviten el retiro de los países del Sistema.

\subsection{Integración económica}

Como se ha señalado en líneas anteriores, la integración económica centroamericana surge entre 1960-1963, con la firma y entrada en vigencia del Tratado General de Integración Económica Centroamericana, el cual crea el Mercado Común Centroamericano (MCCA), que a su vez está orientado a la conformación de la Comunidad Económica Centroamericana (CEC), que tiene como fin impulsar en forma conjunta el desarrollo de Centroamérica y mejorar las condiciones de vida de sus habitantes.

La integración económica tiene enormes ventajas, entre ellas: la creación de grandes mercados, el ensanchamiento de la industria, la promoción del desarrollo económico y la promoción del empleo. Según Ramón Tamames el más extendido de todos los argumentos "es el que se fija en el disfrute de las economías derivadas de la producción en gran escala y del alto grado de especialización que solamente puede ofrecer un amplio mercado interior" (Miranda, 2010: 32).

Así pues, la integración económica tiene como objetivo principal la libre movilización de bienes y servicios, para lo cual es necesario de una armonización de políticas que permitan este fin. De ahí la importancia de contar con órganos debidamente estructurados y facultados en su actuar que se encarguen de elaborar dichas políticas y velar por la ejecución de las mismas.

En términos concretos la integración económica tiene entre sus metas más importantes, las siguientes:

- Estabilización macroeconómica y armonización de políticas

- Perfeccionamiento de la zona de libre comercio centroamericana

- Arancel externo común

- Modernización de las aduanas

- Mejoramiento de la infraestructura

- Atracción de inversiones

- Aseguramiento del financiamiento

- Coordinación de una política conjunta de relaciones comerciales externas.

En virtud de ello es que los países centroamericanos toman la decisión de firmar el Protocolo al Tratado General de Integración Económica Centroamericana (Protocolo de Guatemala) en 1993, el cual define el alcance del proceso de la Integración Económica mediante las siguientes etapas:

- Zona de libre comercio

- Unión aduanera

- Mercado común

- Unión monetaria y económica

- Integración total

Actualmente, si bien es cierto se han realizado esfuerzos para alcanzar una integración económica, como es la exención en el pago de los Derechos arancelarios a la importación (DAI) y exportación, los derechos consulares y demás impuestos y/o cargos vinculados con la importación y exportación de algunos productos originarios; todavía nos encontramos ante una zona de libre comercio imperfecta, ya que aún existen restricciones 
para productos originarios que no gozan de este beneficio; así como otras barreras que imposibilitan una integración económica completa.

\subsection{Integración social, cultural y ambiental}

A pesar de que el elemento social estaba incluido como uno de los ejes de la integración centroamericana, desde 1991 con el Protocolo de Tegucigalpa, el cual contemplaba el establecimiento de cinco subsistemas del SICA: político, económico, social, ambiental y cultural; no fue sino hasta 1994, cuando se logra la incorporación de la temática social de manera más concreta, gracias a la suscripción de la Alianza para el Desarrollo Sostenible de Centroamérica (ALIDES), y posteriormente con la suscripción del Tratado de la Integración Social Centroamericana (TISCA), en 1995.

En ambos documentos se establece el objetivo primordial de la integración social, el cual consiste en garantizar el mejoramiento de la calidad de vida de los pueblos centroamericanos.

Para alcanzar tal fin, se han desarrollado varios programas y proyectos tales como el Programa Regional de Prevención de la Violencia Juvenil en Centroamérica (PREVENIR); el Programa Regional de Seguridad Fronteriza en América Central (SEFRO); el Programa Regional de Seguridad Alimentaria y Nutricional para Centroamérica (PRESANCA); Programa Regional de Sistemas de Información en Seguridad Alimentaria y Nutricional (PRESISAN).

Así mismo, en el sector medioambiental, la Comisión Centroamericana de Ambiente y Desarrollo (CCAD) ha desarrollado proyectos a nivel regional, como el establecimiento de vedas temporales, que buscan la protección de especies animales como langosta y el corredor biológico centroamericano en general. Así mismo, se ha desarrollado una Estrategia Regional Ambiental, con vigencia 2015-2020, que contempla las líneas estratégicas identificadas en las cuales la región debe centrar su foco de acción: cambio climático y gestión del riesgo; bosques, mares y biodiversidad; gestión integral del recurso hídrico; calidad ambiental; comercio y ambiente; así como, mecanismos de financiamiento que permitan a la región contar con los fondos necesarios para implementar acciones orientadas a la conservación y restauración de la zonas degradadas y protección de los ecosistemas remanentes.

No obstante, a pesar de estos esfuerzos los avances sociales logrados no se han podido materializar de manera notable a través de la implementación práctica de políticas públicas regionales.

En general, la consecución de los objetivos de integración social ha sido históricamente complicada debido a diversos obstáculos que se han presentado al tratar de implementar las acciones que permitieran cumplir con los compromisos establecidos. Limitantes debido a conflictos políticos, demasiada amplitud en la visión incorporada a los instrumentos jurídicos, reducida capacidad institucional, la restricción presupuestal y el efecto del Consenso de Washington que hizo que la temática social se relegara a nivel nacional, han dificultado o imposibilitado conseguir mayores avances en los procesos de integración social (CEPAL, 2013:1).

\subsection{Desafíos en la integración centroamericana}

Los países centroamericanos enfrentan ciertas dificultades para fortalecer y alcanzar la completa integración centroamericana. A pesar de los esfuerzos de la subregión por obtener un desarrollo político, económico y social uniforme, todavía no ha logrado crear un modelo de bienestar que permita propiciar un desarrollo integral para alcanzar 
sociedades más equitativas y con niveles reducidos de pobreza.

En la sociedad centroamericana aún persisten problemas vinculados a los elevados niveles de pobreza, desigualdad en los ingresos, problemas de acceso a la educación y a la salud, aumento del mercado laboral informal y alta vulnerabilidad a desastres naturales; y continúa evidenciándose una disparidad en el desarrollo socioeconómico de los países del istmo. "Existe una gran heterogeneidad entre los países que forman parte de la región Centroamericana, que se refleja en importantes diferencias económicas, políticas y sociales que los sitúan en diferentes niveles de desarrollo y que limitan las posibilidades de integración" (CEPAL, 2013: 2).

De igual manera, y como señala Humberto López, Director del Banco Mundial, es necesario "mirar la integración no únicamente como integración comercial, sino también como integración en otras área" ${ }^{2}$. Es preciso desarrollar programas en aras a la integración en otros ámbitos, como salud, educación, ambiental, entre otros.

A esto hay que agregar el hecho que los cambios en el entorno global hacen necesario una renovación del modelo de desarrollo centroamericano; así como, una reforma institucional del Sistema de Integración.

Como manifiesta Víctor Umaña "el sistema institucional actual no responde a las necesidades de la población o lo hace de manera incompleta. La falta de legitimidad de algunos actores del sistema, así como de interlocutores que puedan dialogar y construir rutas comunes, conduce cada vez más hacia una ingobernabilidad, en la que los diferentes grupos de interés velan por sus beneficios o disputan el poder al Estado" ${ }^{3}$.
En este sentido, se hace necesario que órganos como el Parlamento Centroamericano (PARLACEN) y la Corte Centroamericana de Justicia amplíen sus funciones y permitan, desde sus ámbitos de acción, contribuir con un trabajo efectivo y dirigido al bienestar de la población a nivel regional.

Para ello, se hace necesaria de una reforma a los instrumentos jurídicos vigentes, de manera que estos se adecúen al contexto actual de integración regional, y así órganos de vital importancia para el Sistema, como el Comité Ejecutivo del SICA, puedan desempeñar sus funciones de manera eficiente y efectiva, fortaleciendo de esta manera la integración de la región.

Cabe señalar que algunos de estos temas se encuentran comprendidos en la agenda a discutir en la próxima reunión de SICA, que tendrá lugar en julio de este año, cuando Nicaragua asuma la Presidencia Pro Témpore, y que de llegar a un consenso, supondría un gran avance en el fortalecimiento del Sistema y una mayor credibilidad ante la comunidad internacional.

\section{Relación política y económica entre corea y el SICA}

\begin{abstract}
Habiendo abordado en los apartados anteriores el proceso de integración centroamericana, sus diversos ámbitos de integración, situación actual y principales obstáculos; dedicaremos el siguiente apartado para abordar la relación entre la República de Corea y los países centroamericanos miembros de SICA.
\end{abstract}

\subsection{Situación actual}

Desde antes de su incorporación como país observador extrarregional del SICA,

2 Tomado de Revista Bolsa de Noticias, de fecha 05 de Mayo de 2016; pág. 04

3 Artículo de Opinión "Perspectivas de Centroamérica en 2016", por Víctor Umaña. Revista América Economía. Recuperado de http:// www.americaeconomia.com/revista/perspectivas-de-centroamerica-en-2016 
la República de Corea ha manifestado su interés por contribuir al desarrollo integral de Latinoamérica.

Las relaciones económicas entre Corea del Sur y Latinoamérica [incluyendo Centroamérica] entraron en una etapa dinámica en la década de los noventa; desde entonces, existe un progreso constante hasta nuestros días. Este crecimiento fue respaldado por la capacidad competitiva internacional de las industrias manufactureras de Corea del Sur, y la política comercial liberalizadora de los países latinoamericanos (Kim, 2013: 73).

En el caso particular de Centroamérica, Corea, desde el año 1996, ha cooperado con los países de la región, mediante el Foro de Diálogo de Cooperación. En el año 2010, durante la III Reunión de Jefes de Estado y de Gobierno que se celebró entre el SICA y la República de Corea se plasmó el interés de la República de Corea por contribuir aún más a la estabilidad y fortalecimiento de la integración regional, al solicitar su incorporación como observador extrarregional del Sistema.

Su participación en cumbres internacionales (Cumbre Corea-SICA), diálogos políticos de Vice Ministros; la donación de fondos destinados a contribuir al desarrollo de varios países, incluyendo Centroamérica; la gestión de préstamos con bajas tasas de interés; envío de especialistas y voluntarios a los países centroamericanos, entre otros; todo esto con el fin de compartir sus conocimientos, experiencias y tecnología para que los países centroamericanos alcancen un desarrollo integral e igualitario; fortaleciendo la presencia de la subregión en el entorno internacional.

Así mismo, y a la luz del próximo Tratado de Libre Comercio entre la República de Corea y Centroamérica, el cual se encuentra en fase de negociaciones, Corea tiene la disponibilidad de seguir trabajando con los países centroamericanos y con el SICA, a fin de que el Sistema cuente con órganos e instancias con funciones claramente delimitadas, y con el recurso humano y financiero adecuado que le permita llevar a cabo su labor de manera eficiente, y con ello adquirir una mayor credibilidad ante la comunidad internacional; sin detrimento de la cooperación a nivel de país, para fortalecer aún más los lazos de amistad y de cooperación con cada uno de los países centroamericanos.

\subsection{Cooperación en el ámbito internacional}

Para fomentar y consolidar la cooperación con los países del SICA, se han celebrado varios Foros de Diálogo y Cooperación entre Corea y Centroamérica, a partir del cual se han desarrollado congresos y talleres, como el Congreso sobre el "Impulso de la Innovación y el Desarrollo Tecnológico para la Competitividad de la Región"; y el Congreso Regional sobre Desarrollo Rural y Politica Coreana Saemaul Undong, como parte del intercambio de experiencias entre la nación asiática y el bloque regional.

Igualmente, y a través de la Agencia de Cooperación KOICA, la cual funciona como una plataforma a nivel internacional para implementar la concesión de ayuda y cooperación técnica del Programa de Ayuda Oficial al Desarrollo (AOD), en diversas partes del mundo, entre ellas Centroamérica, se ha desarrollado diversos programas con el fin de promover el desarrollo económico y social de los países en vías de desarrollo; y fortalecer los lazos de amistad con los países socios. Así, se ha contado con la visita de varios expertos para compartir experiencias y realizar recomendaciones sobre cómo alcanzar el desarrollo en diversos sectores, como educación, infraestructura, tecnología, comercio, formación técnica, salud, etc.; se han ejecutado programas de capacitación y formación para que estos países cuenten con un capital humano con conocimiento necesario para contribuir al desarrollo de 
los mismos; y también se han desarrollado programas de voluntariado, con el fin de que jóvenes y adultos provenientes de Corea fortalezcan los lazos de amistad y comprensión mutua con los países hermanos en vías de desarrollo; y contribuyan al mejoramiento de la calidad de vida de los habitantes de estos países.

De igual manera, a través de préstamos del Fondo de Cooperación para el Desarrollo Económico (EDCF) se han ejecutado diversos proyectos, como el proyecto de transmisión eléctrica con fuentes renovables, tratamiento de aguas residuales y suministro de agua potable, construcción de carreteras, entre otros; todos en Nicaragua.

Por otro lado, el Gobierno de Corea siente un gran compromiso por profundizar la cooperación hacia sus países hermanos, a fin de que estos al igual que Corea, puedan alcanzar un desarrollo económico, social y tecnológico que les permita salir de la situación de dependencia y pobreza en la que se encuentran. En este sentido, el Gobierno de Corea promueve año con año cursos de capacitación, a fin de que los países en vías de desarrollo (incluido Centroamérica) tengan acceso a conocimiento técnico avanzado en diversas temáticas y sectores (TIC, agricultura y ganadería, electrónica, industria automotriz, etc.); así como formación académica especializada, a través de los estudios de maestrías y doctorados que se ofrecen de Gobierno a Gobierno.

Así mismo, resulta oportuno mencionar el compromiso, que ha adquirido la República de Corea, de aumentar gradualmente el volumen de los fondos reservados para su Programa de Ayuda Oficial al Desarrollo (AOD). En concreto, se espera que el volumen del fondo de la AOD equivaldrá al $0.2 \%$ del ingreso nacional bruto (INB) de
Corea en 2020, un incremento con respecto al casi $0.13 \%$ registrado en $2013^{4}$.

\subsection{Inversión de Corea en los países del SICA}

Corea del Sur requiere de la economía de Latinoamérica [incluyendo la economía centroamericana] en varias dimensiones; en primer lugar, Latinoamérica le sirve como proveedor de energía y minerales estratégicos como el petróleo crudo, hierro, cobre, entre otros; segundo, es abastecedor de recursos alimenticios como agropecuarios y de pescadería; tercero, es mercado de consumidores de productos y de insumos industriales; cuarto, es una base de manufactura para mercado local o plataforma para penetrar en los mercados [más grandes como Estados Unidos y la Unión Europea] , y por último, es un mercado de infraestructura, incluyendo construcción de plantas de refinería o generación eléctrica (Kim, 2013: 78).

Para el autor, la relación económica entre Corea y Latinoamérica se caracteriza por ser un comercio "inter-industrial", en virtud del cual "Corea realiza importaciones de productos primarios y minerales o productos agrícolas; exporta los productos secundarios y terciarios o manufacturados, así como servicios" (Kim, 2013: 78). Este tipo de comercio inter-industrial "contribuye a la competencia económica de Latinoamérica, al promover la industrialización" (Kim, 2013: 78).

No obstante, se debe trabajar por la tecnificación de los países de la región, a fin de que estos cuenten con una industria manufacturera que les permita no sólo exportar materia prima a las economías avanzadas; sino también exportar productos manufacturados; para lo cual es necesario

4 Cifras tomadas de artículo electrónico La ayuda oficial al Desarrollo de Corea del Sur alcanzará un 0,2 por ciento del INB en 2020. Recuperado de http://spanish.yonhapnews.co.kr/national/2015/11/10/0300000000ASP20151110002600883.HTML 
contar con la tecnología adecuada y el recurso humano con conocimiento que permita a estos países alcanzar la industrialización requerida, siendo de gran valor la transferencia de tecnología que Corea realiza hacia estos países a través de diversos Foros, talleres y seminarios, como el Foro de Cooperación de América Latina y El Caribe (FOCALAE).

Según datos del Banco de Desarrollo de América Latina (CAF), Corea y Centroamérica han ampliado sus intercambios comerciales a un ritmo promedio del $16 \%$ anual en sus últimos años. En el año 2014, el monto comercial que Corea exportó a los seis países de América Central fue de U\$ 3.8 billones de dólares y la importación de productos provenientes de la región centroamericana fue de U\$ 1.2 billones de dólares; habiendo alcanzado en ese año una inversión de U\$3.86 billones de dólares.

En la actualidad, en cada país de América Central existe alrededor de 30-100 PYMES coreanas principalmente en la industria textil. Pero recientemente, está aumentando las empresas que se dedican a los sectores de construcción, transmisión eléctrica, energías renovables etc. Estas empresas coreanas establecidas en Centroamérica aportan al desarrollo de la región al fortalecer y diversificar sus áreas de inversión.

\section{Conclusiones}

A partir de lo expuesto se puede concluir que la integración centroamericana ha sufrido por varios intentos, desde la época colonial hasta el presente, en alcanzar el estado de integración actual.

Así mismo, el proceso de integración centroamericana, al igual que en otras regiones, es un proceso vinculado estrechamente con la voluntad de los países miembros. Su avance o estancamiento depende directamente de la anuencia que tengan estos en profundizar el proceso de integración, lo cual se evidencia en la elaboración y aplicación de políticas para tal fin.

Si bien se han logrado algunos avances, todavía subsisten barreras (esencialmente de carácter ideológico, político e institucional) que impiden la completa integración. Es necesario realizar una reforma a los instrumentos jurídicos, en particular al Protocolo de Tegucigalpa, a fin de contar con órganos e instituciones regionales que lleven a cabo sus funciones con eficacia.

Los países miembros deben adoptar políticas, a fin de alcanzar similares niveles en áreas como educación, seguridad ciudadana, comercio, economía, medio ambiente, entre otros, que hagan propicia la integración.

La República de Corea debe desarrollar estrategias que permitan el acercamiento $\mathrm{y}$ fortalecimiento de las relaciones con el SICA en general, y sus países miembros en particular. En este sentido, el intercambio de conocimientos en el área de Ciencias y Tecnología es fundamental, a fin de mejorar el nivel socio-económico de la región.

En este sentido, en primer lugar, es necesario reconocer el avance que ha tenido la región de América Central para aceptarlos como verdaderos socios estratégicos. Los países centroamericanos han constituido un aliado político que brindó apoyo al Gobierno de la República de Corea. Corea necesita fortalecer esta alianza para el desarrollo de las relaciones amistosas tradicionales, promoviendo la democracia y la economía de mercado en América Central; y uniendo esfuerzos en la lucha contra las drogas y el terrorismo, que afectan el campo de la integración, incluidos los derechos humanos.

En segundo lugar, la República de Corea puede contribuir al desarrollo de la región de América Central, mediante la mejora de la Ciencia y Tecnología de estos países, a fin de 
que puedan efectuar una reforma estructural en su industria, que les permita ingresar al mercado exportador con mayor solidez y competitividad. Este desarrollo tecnológico abriría las puertas para construir nuevas alianzas en aras de compartir los beneficios económicos mutuos en futuro, al tiempo que contribuye al desarrollo económico y social de toda la región de América Central.

En tercer lugar, y teniendo en cuenta el valor y la ubicación estratégica de América Central, necesaria para acceder a un mercado más amplio, se hace necesario desarrollar una estrategia regional. Para ello es necesario el establecimiento de un "Plan de cooperación para el desarrollo a largo plazo del SICA", el cual comprenda a su vez un fondo separado destinado a proporcionar una cooperación planificada y sistemática a nivel local.

\section{Referencias bibliográficas}

Asamblea Nacional de la República de Nicaragua. Dirección de Relaciones Internacionales Parlamentarias. (2014). Análisis al Protocolo de Tegucigalpa a la Carta de la Organización de Estados Centroamericanos (ODECA). Managua, Nicaragua.

Bulmer Thomas, V. (1998). Integración Regional en Centroamérica. $1^{\text {a }}$ Edición, Editorial FLACSO-SSRC. San José, Costa Rica. 348 págs.

Cardenal, R. (1996). Manual de Historia de Centroamérica. UCA Editores, El Salvador.
CEPAL. (2013). Integración Social en Centroamérica. Situación al 2012, tendencias y propuestas. México D.F. Recuperado de

Kim, Won-Ho. (2013). La situación actual de las relaciones económicas de Corea del Sur con Latinoamérica: el nuevo paradigma y los retos. PORTES. Revista mexicana de estudio sobre La Cuenca del Pacífico. Volumen 7; Número 14. Julio/Diciembre 2013. Colima, México. Recuperado de http:// revistasacademicas.ucol.mx/index. $\mathrm{php} /$ portes/article/view/179/152

Miranda, O. (2010). Derecho Comunitario de América Central. $1^{\mathrm{a}}$ ed. Universidad Centroamericana. Facultad de Ciencias Jurídicas. Managua, Nicaragua.

OIT. (1998). El Proceso de Integración Centroamericana en perspectiva. Recuperado de http://www.ilo.org/ public/spanish/region/ampro/ mdtsanjose/worker/integ_ca/final. htm

Protocolo de Tegucigalpa a la Carta de Organización de los Estados Centroamericanos (ODECA), del 13 de diciembre de 1991

Protocolo al Tratado General de Integración Económica Centroamericana (Protocolo de Guatemala), del 29 de octubre de 1993

Reglamento de los Actos Normativos del Sistema de Integración Centroamericana, del 01 de diciembre de 2005 